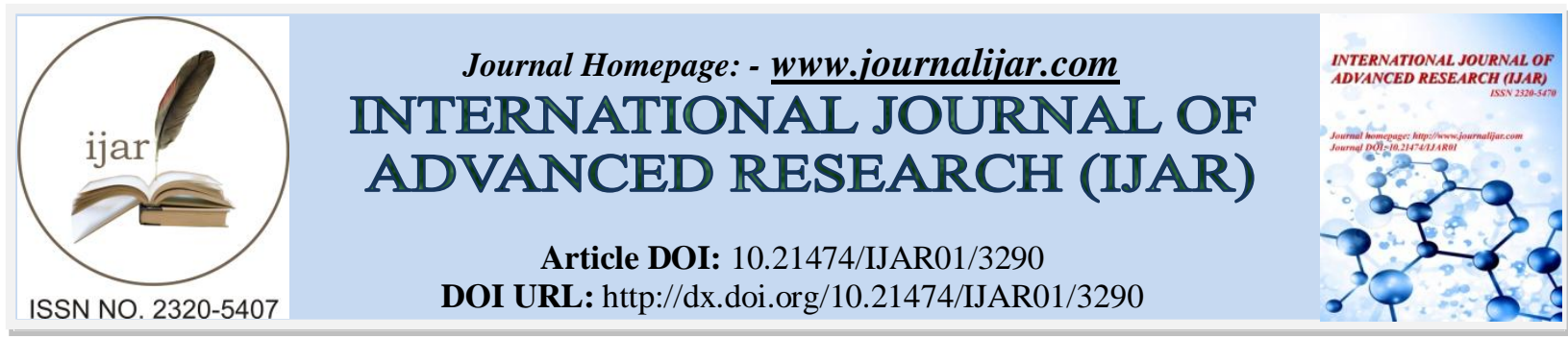

RESEARCH ARTICLE

\title{
NITRITE OXIDE DETECTION UTILIZING THE GRIESS ASSAY: ELUCIDATING INTERFERING FACTORS FOR IN VITRO APPLICATION.
}

Turki Alsharekh and Faisal Albaqami.

\section{Manuscript Info}

Manuscript History

Received: 06 December 2016

Final Accepted: 12 January 2017

Published: February 2017

\begin{abstract}
Nitrite Oxide (NO) is a molecular mediator of many physiological processes including: Vasodilation, Inflammation, Immunity\& Neurotransmission.But we can't measure Nitrite Oxide formation Directly by Griess assay so we use one of its stable non-volatile breakdown products: the Nitrite ( NO2-).In our paper we look into different factors that may change the Nitrite Oxide Quantification in Griess Assay reaction for in-vitro application including:Culture Media(DMEM , RPMI , MEM and RPMI-F12) \&Anticoagulants(Heparin And Enoxaparin). This is done by comparing the results from a control Griess Assay against a Griess Assay with either Culture media or Anticoagulant. From the results we conclude that a) Culture Media produce similar results to Calibration curve. B) Griess assay is effected by Heparin and Enoxoparin.
\end{abstract}

Copy Right, IJAR, 2017,. All rights reserved.

\section{Introduction:-}

Nitrite Oxide (NO) is a molecular mediator of many physiological processes including: Vasodilation, Inflammation, Immunity\& Neurotransmission. A number of methods exist for measuring Nitrite Oxide in biological systems; one of these is Griess Assay. But we can't measure Nitrite Oxide formation Directly by Griess assay so we use one of its stable non-volatile breakdown products: the Nitrite ( NO2-). [1]

The Griess assay is a chemical analytical test that is used to measure the levels of Nitrite. The Assay based on a chemical reaction which uses sulfanilamide and N-1-napthylethylenediamine dihydrochloride under acidic conditions. Sulfanilic acid is quantitatively converted to a diazonium salt by reaction with nitrite in acid solution. The diazonium salt is then coupled to NED, forming an azo dye that can be spectrophotometrically quantitated based on its absorbance at $540 \mathrm{~nm}$. Thus it detects NO2- in a several biological and experimental liquids such as plasma, serum, urine and tissue culture medium. [2] 

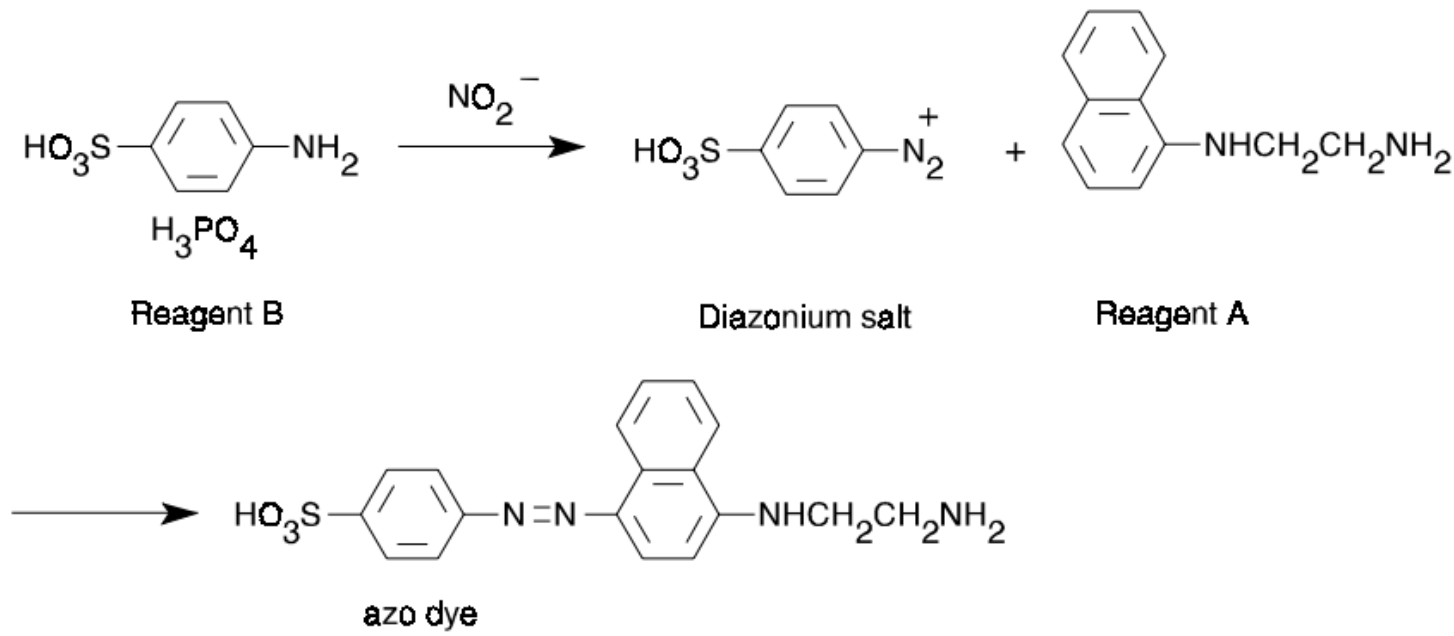

Figure.1:- Principle of Nitrite Quantitation using Griess reaction. [1]

\section{Objective:-}

In our paper we shall look into different factors that may change the Nitrite Oxide Quantification in Griess Assay reaction for in-vitro application including:

1. Culture Media:DMEM, RPMI, MEM and RPMI-F12.

2. Anticoagulants including Heparin And Enoxaparin.

\section{Method:-}

Heparin and Enoxaparin (Low Molecular Weight Heparin ) will be used to determine if these have any effect on Griess assay.

To do our projects first we need to make a couple of solutions:-

Stock solution A ( $50 \mathrm{ml})$ which is composed of :

$1.0 .4 \%$ NED.(200mg in 50ml).

2. $4 \%$ Sulphanilamide. $(2 \mathrm{~g}$ in $50 \mathrm{ml})$.

3. $10 \%$ Phosphoric acid ( $6 \mathrm{ml}$ of $85 \%$ stock )

\section{Solution B ( 15ml ) which is composed of:-}

4. $3 \mathrm{ml}$ Stock Solution A.

5. $12 \mathrm{ml}$ MilliQ water.

Method for Traditional Griess Assay Curve:-

1. We Added $100 \mu \mathrm{l}$ of $5 \mathrm{mM}$ Sodium Nitrite to all wells of column 1 of a 96 well-plate.

2. Then we Added $100 \mu \mathrm{l}$ of MilliQ water media to each of all remaining wells.

3. After that we serially dilute (1:2) sodium Nitrite across the plate.

4. Add $100 \mu \mathrm{l}$ Solution B to all wells on the 96-well plate.

5. Finally we measured the absorbance at $\lambda \mathrm{abs}=540 \mathrm{~nm}$ ( or $570 \mathrm{~nm}$ ) by using a plate reader .

\section{Variations:-}

Replace MilliQ water and add Culture Media / Heparin solution / Low Molecular weight Heparin in its place to detect changes. 


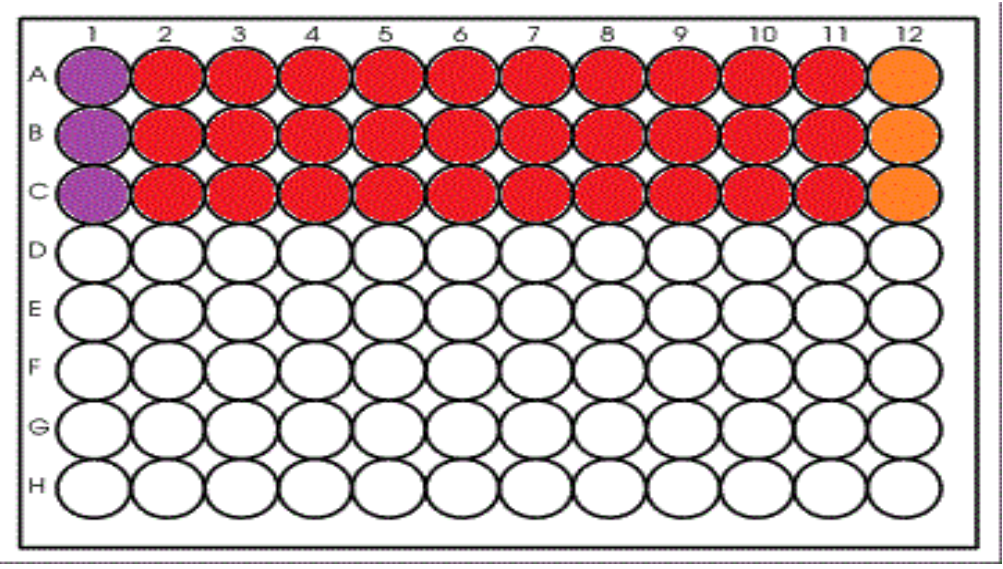

Figure 2:- Representation of the 96 well-plate. Left is the highest concentration of Nitrate serially diluted to the right.Three rows were used and averaged to maintain lower chance error.[3]

For the results we shall compare culture media, heparin solution and enoxaparin solution to the Traditional Curve which is used as control.

\section{Results:-}

We will use graphs \& Linear equations to represent the results. First, we will look at Traditional Curve which will be compared to the rest of the results. Then we will look at culture media $\&$ heparin results.

Initial results on Traditional curve showed that the absorbance on the spectrophotometer is not accurate at higher concentrations ( $1.5 \mathrm{uM}$ to be specific) and thus we have lowered the Concentration to 0.3125 to be the highest concentration of Nitrate to maintain accuracy.

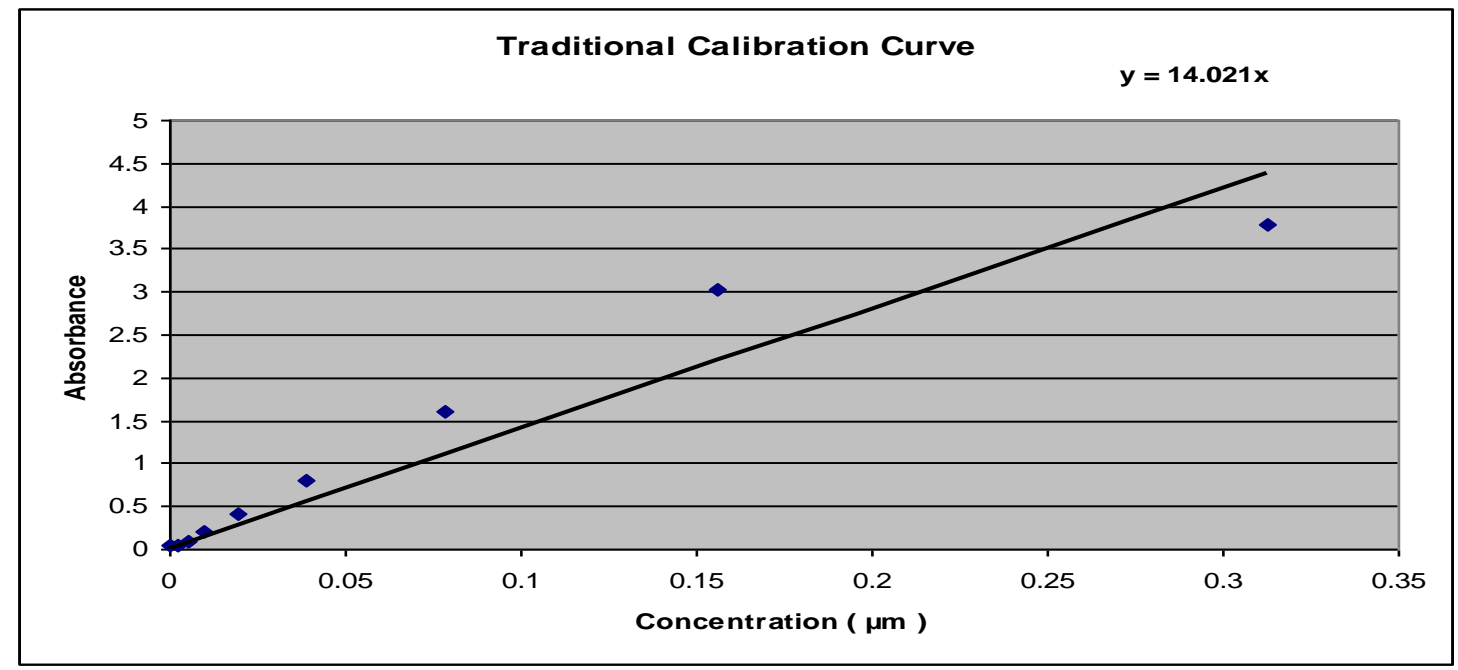

Graph.1:- Traditional Calibration Curve, which will be used as control. 

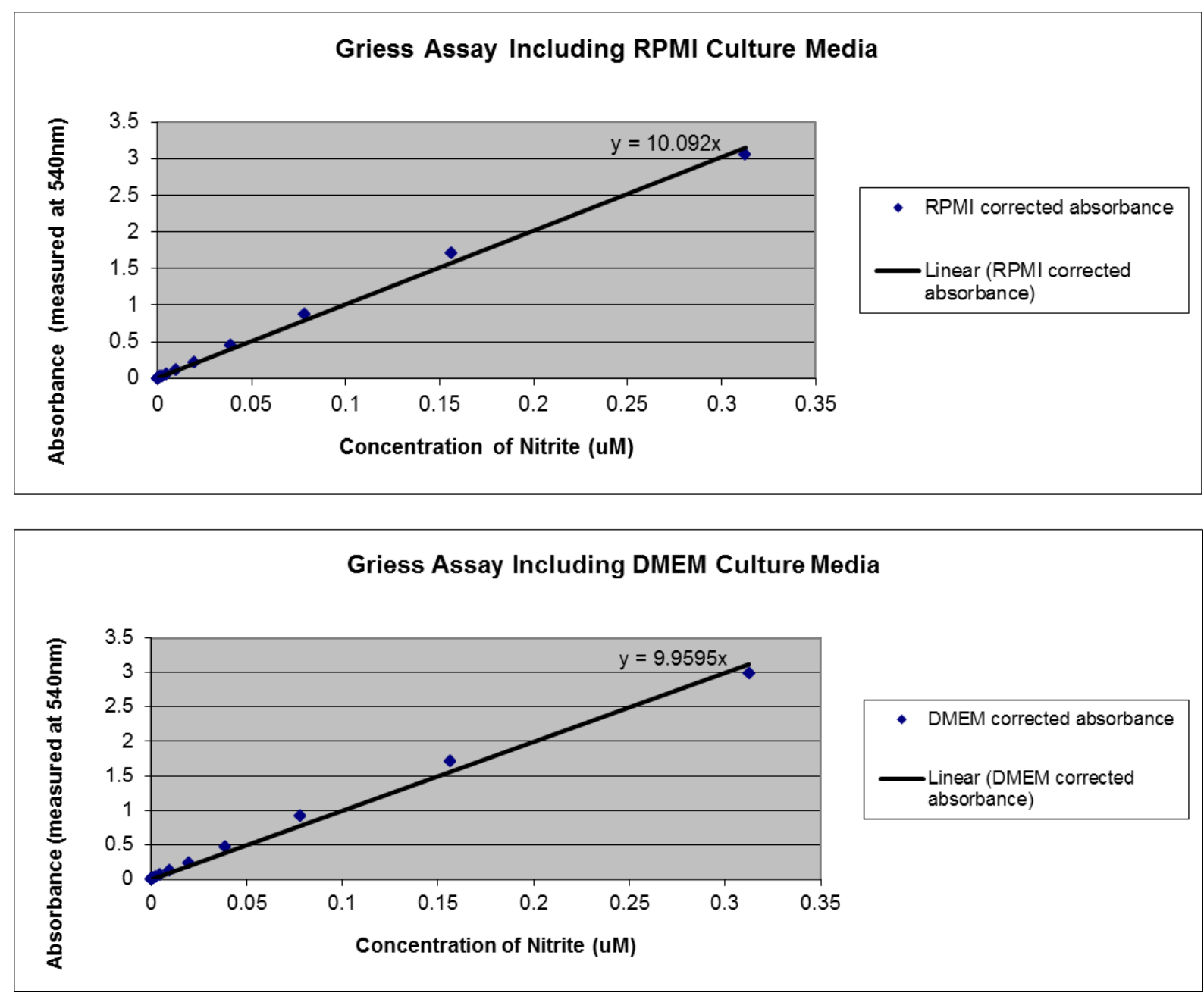

Graph. 2 \& 3:- RPMI and DMEM Culture Media, There is small difference between them and Calibration Curve. 

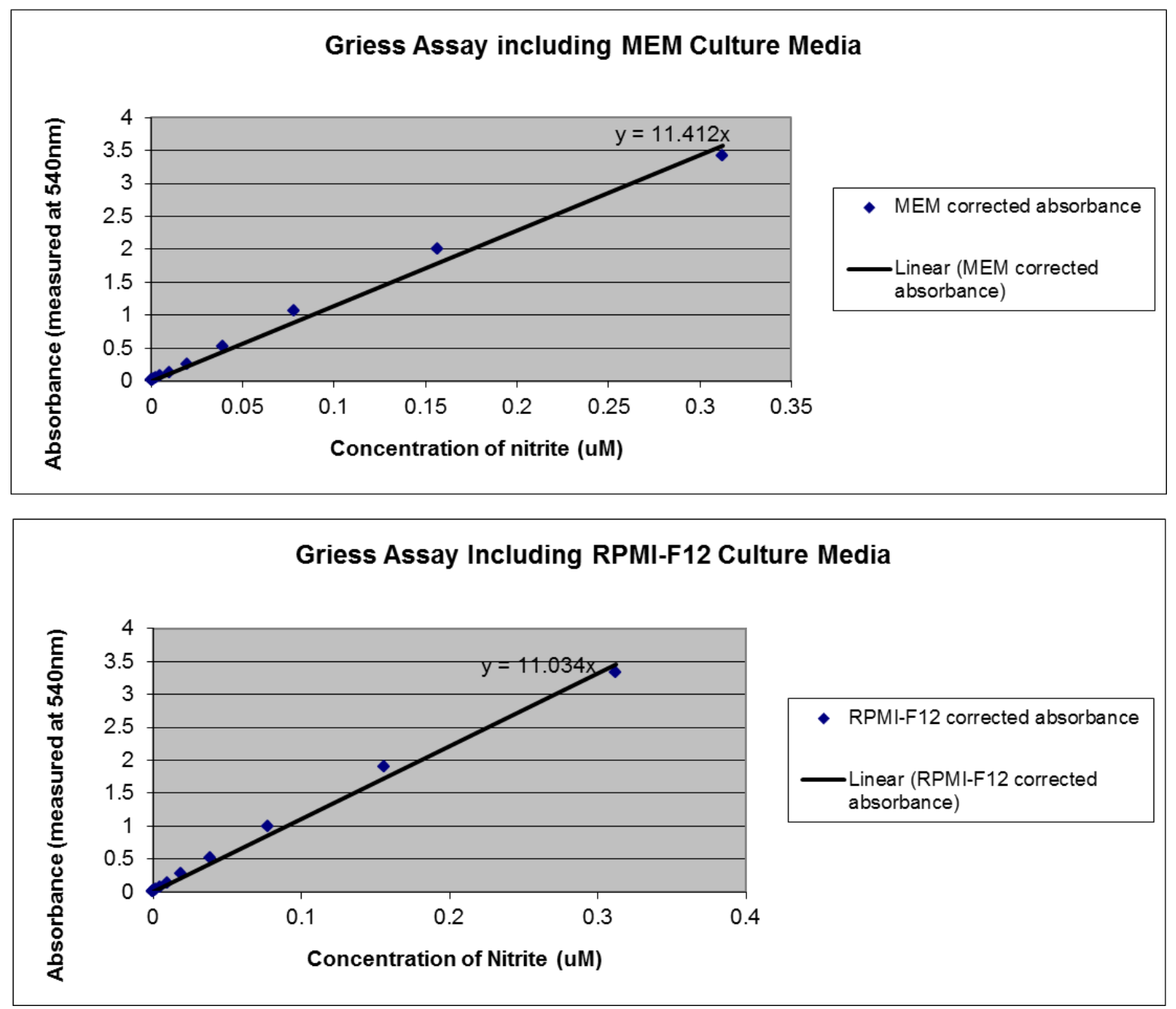

Graph 4 \& 5:- RPMI-F12 and MEM Culture Media, still similar to previous results.

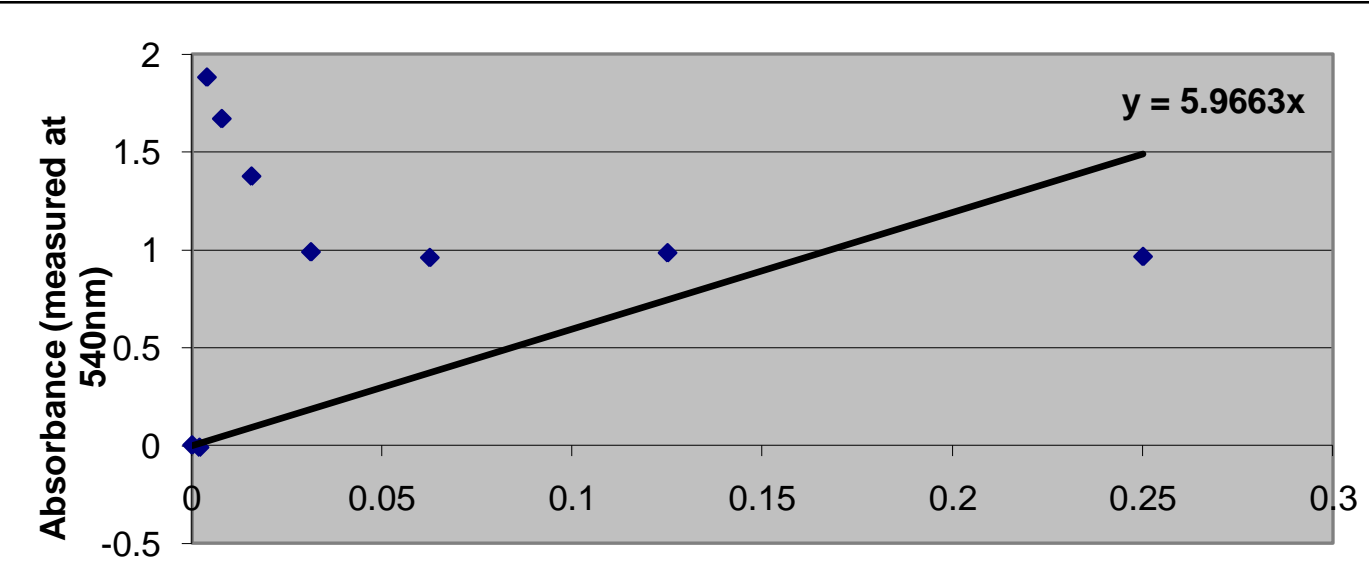

Concentration ( UM) 


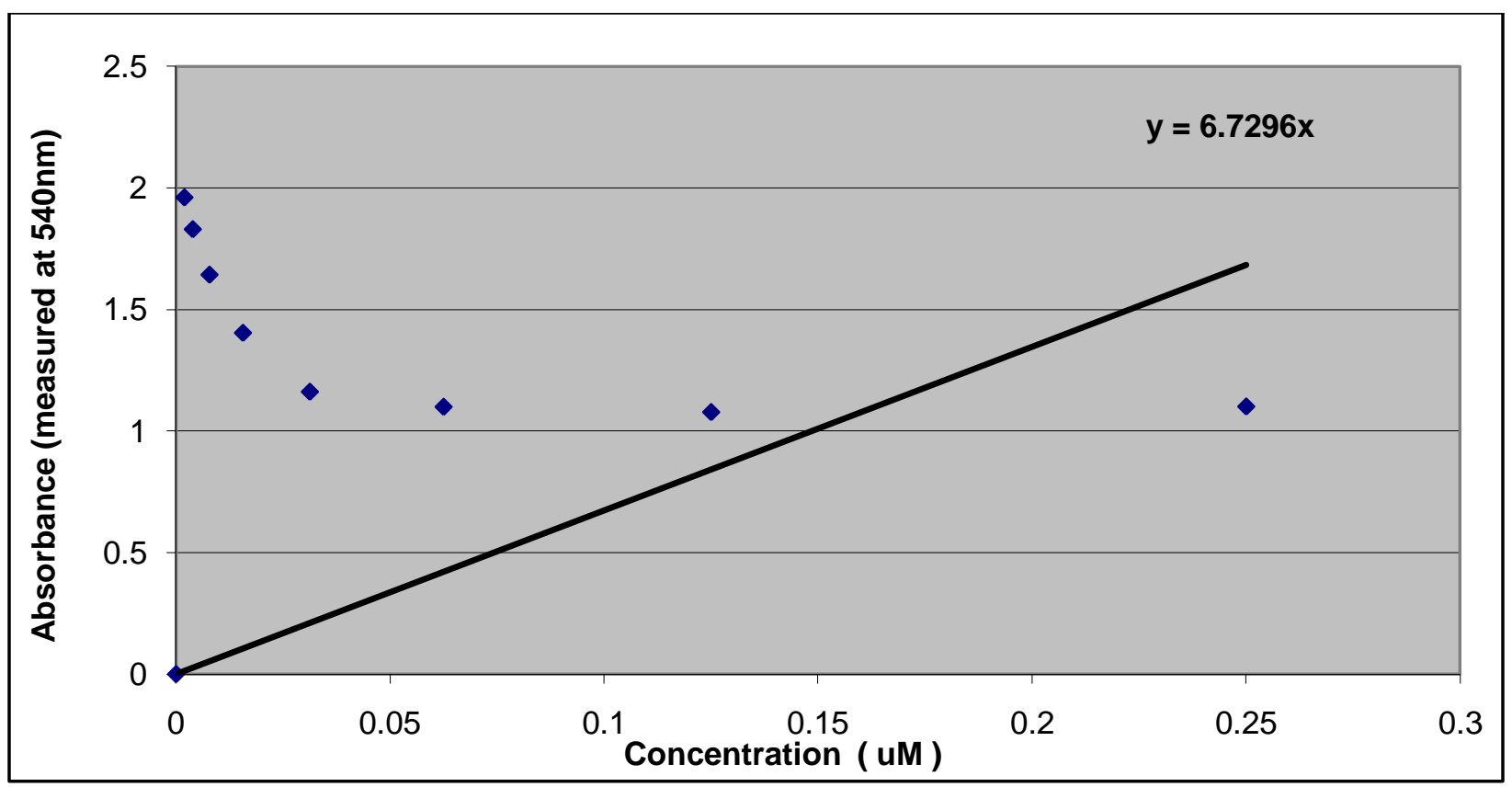

Graph. 6 \& 7:0- Heparin and Enoxaparin, from analysing the graphs and trendlineswe found that Griess Assay is prone to interferences with Heparin and Enoxaparin and gives altered results.

\section{Conclusion:-}

From the results we conclude that a) Culture Media produce similar results to Calibration curve. B) Griess assay is effected by Heparin and Enoxoparinand gives altered results.

\section{Sources:-}

1. Griess Reagent Kit for Nitrite Determination (G-7921) - Molecular Probes.

2. Griess Reagent SystemINSTRUCTIONS FOR USE OF PRODUCT G2930.

3. Figure is made by Authors. 